\title{
Optimal Design of a EWMA Chart to Monitor the Normal Process Mean
}

\author{
Jaeheon Lee ${ }^{1}$ \\ ${ }^{1}$ Department of Applied Statistics, Chung-Ang University
}

(Received March 2, 2012; Revised May 3, 2012; Accepted June 5, 2012)

\begin{abstract}
EWMA(exponentially weighted moving average) charts and CUSUM(cumulative sum) charts are very effective to detect small shifts in the process mean. These charts have some control-chart parameters that allow the charts and be tuned and be more sensitive to certain shifts. The EWMA chart requires users to specify the value of a smoothing parameter, which can also be designed for the size of the mean shift. However, the size of the mean shift that occurs in applications is usually unknown and EWMA charts can perform poorly when the actual size of the mean shift is significantly different from the assumed size. In this paper, we propose the design procedure to find the optimal smoothing parameter of the EWMA chart when the size of the mean shift is unknown.
\end{abstract}

Keywords: Design of a control chart, average run length, statistical process control, EWMA chart.

\section{1. 서론}

공정의 변화를 탐지하는 통계적 공정관리에서 가장 빈번하게 발생하는 문제는 관측값들이 서로 독립인 정규분포를 따르는 공정에서 평균의 변화를 탐지하는 것이다. 전통적으로 사용하는 Shewhart 관리도 는 공정 평균의 큰 변화 탐지에는 효율적이지만, 작은 변화 탐지에는 효율적이지 못하다. 반면에 Page (1954)가 제안한 CUSUM 관리도와 Roberts (1959)가 제안한 EWMA 관리도는 공정 평균의 작은 변 화 탐지에 효율적이라는 사실이 잘 알려져 있다.

CUSUM 관리도와 EWMA 관리도는 Shewhart 관리도에 비하여 상대적으로 그 효율이 우수하다는 장 점이 있지만, 관리한계(control limit) 외에 사전에 설정해야 하는 모수를 가지고 있다는 단점이 있다. 먼저 CUSUM 관리도에서는 탐지하기를 원하는 변화의 양 또는 참고값(reference value)을 지정해야 한 다. 일반적으로, 예상하는 공정의 표준화된 변화량을 2 로 나눈 값을 참고값으로 사용하고 있다. 그러나 공정의 변화량이 미리 설정한 값과 다르게 발생한 경우 CUSUM 관리도의 효율은 떨어지게 된다. 보 통 공정의 변화량을 미리 예측하기가 쉽지 않기 때문에, 이와 같은 문제점을 해결하는 방법에 대한 연구 가 진행되어 왔다. Shewhart 관리도와 병행하거나 또는 몇 개의 CUSUM 관리도를 병행하는 것이 쉽

This research was supported by Basic Science Research Program through the National Research Foundation of Korea (NRF) funded by the Ministry of Education, Science and Technology (2010-0009571).

${ }^{1}$ Corresponding author: Professor, Department of Applied Statistics, Chung-Ang University, 221 HeukseokDong, Dongjak-Gu, Seoul 156-756, Korea. E-mail: jaeheon@cau.ac.kr 
게 고려할 수 있는 방법이다 (Lucas, 1982; Zhao 등, 2005). 이와는 달리 공정의 탐지를 진행하면서 매 시점 마다 참고값을 변화시키는 방법을 고려할 수도 있다 (Spark, 2000; Shu와 Jiang, 2006; Jiang 등, 2008). 최근 Ryu 등 (2010)은 평균 변화량을 모르는 경우 CUSUM 관리도의 최적 설계에 관한 절차를 제안하였다. 그들은 최적화를 위하여 3 가지의 측도를 고려하였고, 변화량에 대한 여러 가지 사전분포에 대하여 최적의 참고값을 설정하는 절차를 제안한 것이다.

유사하게 EWMA 관리도에서는 평활모수(smoothing parameter) 또는 가중치(weight)를 미리 설정해 야 한다. 공정의 작은 변화를 탐지할 경우에는 작은 평활모수 값을 사용하고, 큰 변화를 탐지할 경우에 는 큰 평활모수 값을 사용하는 것이 효율적이라고 알려져 있다. 그러나 위에서 언급한 바와 같이 공정의 변화량을 예측하기는 쉽지 않기 때문에, 평활모수의 설정 또한 용이하지가 않게 된다. CUSUM 관리도 의 경우와 유사하게 EWMA 관리도에서도 Shewhart 관리도 또는 여러 EWMA 관리도를 병행하거나 매 시점 마다 평활상수를 변화시키는 방법을 고려할 수 있다 (Capizzi와 Masarotto, 2003; Reynolds와 Stoumbos, 2006).

이 논문의 목적은 평균 변화량을 예상하기 어려운 경우 EWMA 관리도에서 최적의 평활모수 설정에 관 한 절차를 제안하는데 있다. 또한 여러 가지 주어진 조건에서 설정된 최적의 평활모수에 대한 경향과 특 징을 알아보고자 한다.

\section{EWMA 관리도}

관측값들이 서로 독립이고 $N\left(\mu_{0}, \sigma_{0}^{2}\right)$ 를 따르는 확률변수인 공정을 고려해 보자. 매 시점마다 크기가 $n$ 인 표본을 관측하고 시점 $t$ 에서 계산한 표본평균을 $\bar{X}_{t}$ 라 할 때, 공정 평균의 변화를 탐지하는 EWMA 관리도의 통계량은

$$
E_{t}=\lambda \bar{X}_{t}+(1-\lambda) E_{t-1}
$$

이 된다. 여기서 $E_{0}=\mu_{0}$ 이고 $\lambda$ 는 $0<\lambda \leq 1$ 인 평활모수이며, $\lambda=1$ 인 경우에는 Shewhart의 $\bar{X}$ 관리 도가 된다. 공정 평균이 $\mu_{0}$ 에서 $\mu\left(\neq \mu_{0}\right)$ 로 변화하는지를 탐지하는 $\mathrm{EWMA}$ 관리도 절차는 $E_{t}$ 의 값이 관리한계인

$$
\mu_{0} \pm c \sqrt{\frac{\lambda}{2-\lambda}} \frac{\sigma_{0}}{\sqrt{n}}
$$

를 벗어날 경우 이상상태의 신호를 주는 것이다. $c$ 는 일반적으로 관리상태에서의 평균런길이(average run length; $\mathrm{ARL}$ ), 즉 $\mathrm{ARL}_{0}$ 가 주어진 값을 만족하도록 설정하고 있다. 이 논문에서는 일반성을 잃지 않고 $\mu_{0}=0$ 이고 $\sigma_{0}=1$ 임을 가정하고, 표본의 크기는 $n=1$ 인 경우를 고려하고자 한다. Reynolsd와 Stoumbos $(2004 \mathrm{a}, 2004 \mathrm{~b})$ 는 CUSUM 관리도와 EWMA 관리도에서 $n=1$ 인 경우의 효율이 전반적으 로 제일 좋다는 연구 결과를 발표한 바 있다.

일반적으로 관리도의 효율은 평균런길이인 $\mathrm{ARL}$ 로 측정하고 있다. 물론 이와 유사한 개념인 신호까지 의 평균 시간을 나타내는 ATS(average time to signal)와 신호까지의 평균 관측값의 수를 나타내는 ANOS(average number of observations to signal)를 사용할 수 있다. EWMA 관리도에서 효율의 측 도인 $\mathrm{ARL}$ 을 계산하는 여러 가지 방법들이 제안되어 있으나, 이 논문에서는 가장 많이 사용하고 있는 다음과 같은 방법으로 $\mathrm{ARL}$ 을 계산하고자 한다. $L(u)$ 를 초기치가 $E_{0}=u$ 인 $\mathrm{EWMA}$ 관리도의 $\mathrm{ARL}$ 이 라 할 때, Crowder (1987a)는 $L(u)$ 가

$$
L(u)=1+\frac{1}{\lambda} \int_{\mathrm{LCL}}^{\mathrm{UCL}} L(x) f\left(\frac{x-(1-\lambda) u}{\lambda}\right) d x
$$


와 같이 표현된다는 것을 보였다. 여기서 UCL과 LCL은 각각 식 (2.1)에 기술한 관리상한(upper control limit)과 관리하한(lower control limit)을 나타내며, $f(x)$ 는 표본평균 $\bar{X}_{t}$ 의 확률밀도함수를 나타낸 다. 식 $(2.2)$ 에 표현된 적분방정식은 Fredholm 적분방정식의 형태로서 수치해석적인 방법으로 $L(u)$ 를 계산할 수 있으며, 이 방법으로 ARL을 계산하는 프로그램은 Crowder (1987b)를 참고할 수 있다.

\section{3. 최적의 평활모수 선정}

앞에서 언급한 바와 같이 관리도의 효율은 일반적으로 $\mathrm{ARL}$ 을 이용하여 나타내고 있다. 관리상태에서 의 $\mathrm{ARL}$ 을 특정한 값으로 고정시킨 후, 이상상태에서의 $\mathrm{ARL}$ 이 상대적으로 작은 값을 가질 때 관리도 의 효율이 좋다고 할 수 있다. 그러나 관리도를 비교할 때 여러 가지의 변화량에 대하여 ARL만을 이용 하여 효율을 측정하기에는 부족한 점이 있다. 예를 들어 두 관리도 $\mathrm{A}$ 와 $\mathrm{B}$ 를 비교할 때, 공정 평균의 작 은 변화에 대해서는 관리도 $\mathrm{A}$ 의 효율이 더 좋았지만 큰 변화에 대해서는 관리도 $\mathrm{B}$ 의 효율이 더 좋다고 가정해 보자. 공정 평균이 크게 변했을 때 이를 늦게 탐지하는 것이 작게 변했을 때에 비하여 상대적으 로 더 큰 손실을 가져올 수 있기 때문에, 작은 변화량에 대한 $\mathrm{ARL}$ 의 차이와 큰 변화량에 대한 $\mathrm{ARL}$ 의 차이를 동등한 기준으로 비교하는 것은 공정하지 못한 결과를 가져올 수 있다.

Reynolds와 Lou (2010)는 정규분포를 따르는 공정의 평균을 탐지하기 위하여 GLR(generalizd likelihood ratio) 관리도의 사용을 제안하고 관리도의 효율을 측정하기 위하여 ATS와 함께 $\mathrm{EQL}(\mathrm{extra}$ quadratic loss)이라는 측도를 사용하였다. 이것은 특정한 크기의 변화로 인하여 발생하는 손실을 이차 손실함수(quadratic loss function)의 개념을 이용하여 표현한 것으로 표준화된 이차 손실은

$$
E\left[\left(\frac{X_{i}-\mu_{0}}{\sigma_{0}}\right)^{2}\right]=1+\delta^{2}
$$

로 정의할 수 있는데, 여기서 $X_{i}$ 는 공정의 관측값이고 $\delta$ 는 $\delta=\left(\mu-\mu_{0}\right) / \sigma_{0}$ 로 정의되는 표준화된 변화 량을 나타낸다. 공정이 관리상태일 때, 즉 $\mu=\mu_{0}$ 일 때 표준화된 이차 손실은 1 이므로, 공정 평균의 변 화로 인한 추가적인 손실은 $\delta^{2}$ 이 된다. 따라서 변화량 $\delta$ 로 인한 총손실의 기대값인 $\mathrm{EQL}(\delta)$ 는

$$
\operatorname{EQL}(\delta)=E\left[\sum_{i=1}^{\mathrm{RL}(\delta)} \delta^{2}\right]=\delta^{2} \operatorname{ARL}(\delta)
$$

가 된다. 여기서 $\operatorname{RL}(\delta)$ 와 $\operatorname{ARL}(\delta)$ 는 각각 변화량 $\delta$ 에 대한 런길이와 평균런길이를 나타낸다. 여러 가 지의 변화량 $\delta$ 를 동시에 고려하기 위해서는 $\delta$ 의 발생 확률에 대한 사전분포(prior distribution)가 필요 하다. $\delta$ 의 사전분포를 $\pi(\delta)$ 라고 할 때, 모든 변화량을 고려한 $\mathrm{EQL}$ 은

$$
\mathrm{EQL}=\int \operatorname{EQL}(\delta) \pi(\delta) d \delta
$$

로 정의할 수 있다. Ryu 등 (2010)도 CUSUM 관리도의 최적 설계를 위하여 이와 비슷한 측도를 사용 한 바 있다.

$\mathrm{EWMA}$ 관리도의 최적 설계는 식 (3.1)에 정의된 $\mathrm{EQL}$ 을 최소로 하는 평활모수 $\lambda$ 를 찾는 것과 동일 하다. 이를 위하여 사전분포로는 감마분포(gamma distribution)와 균등분포(uniform distribution)를 고려하였다. $G(a, b)$ 는 형상모수(shape parameter)가 $a$ 이고 척도모수(scale parameter)가 $b$ 인 감마 분포를 나타내며, $U(c, d)$ 는 구간 $[c, d]$ 에서 정의되는 균등분포를 나타내기로 한다. 이 논문에서는 Reynolds와 Lou (2010)와 같이 변화량 $\delta$ 의 범위로 $[0.25,7]$ 을 고려하였고, $G(1,1), G(1,2), G(1,3)$, $G(2,1), G(2,2)$, 그리고 $G(3,1)$ 의 6 가지 감마분포와 $U(0.25,7)$ 의 1 가지 균등분포를 사용하였다. 모 


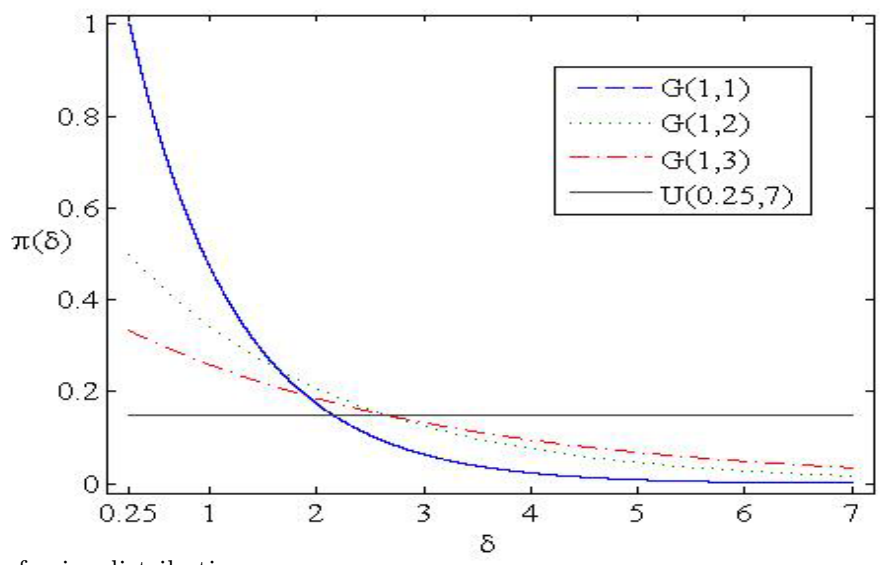

Figure 3.1. pdf of prior distributions

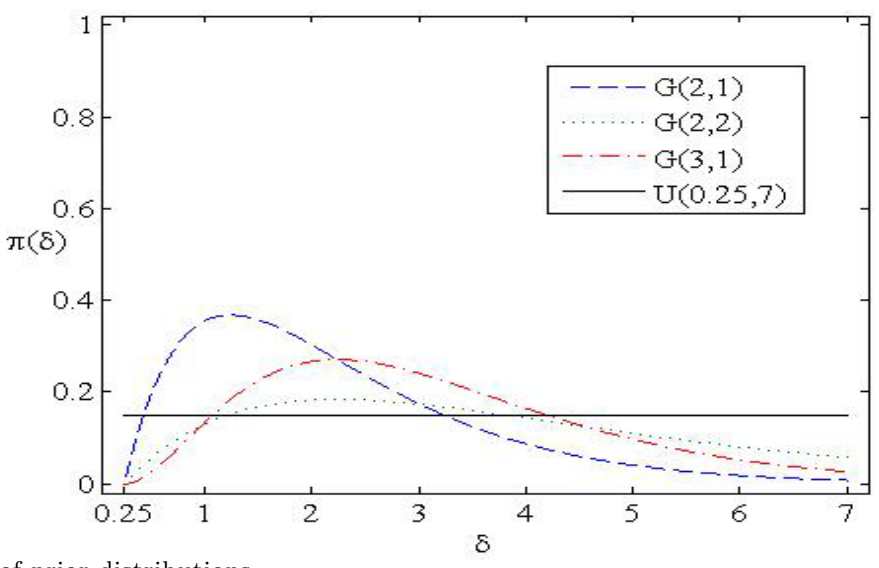

Figure 3.2. pdf of prior distributions

든 감마분포는 6.75 에서 절단하여 $[0,6.75]$ 의 범위를 $[0.25,7]$ 로 변환시켜 사용하였다. 모든 사전분포의 확률밀도함수를 Figure 3.1과 Figure 3.2에 나타내었고, 이와 같은 사전분포를 가정할 경우 식 (3.1)의 $\mathrm{EQL}$ 을 최소로 하는 최적의 평활모수의 값, 이 모수를 사용할 경우 식 (2.1)의 관리한계에서 $c$ 값, 그리 고 이때의 $\mathrm{EQL}$ 값을 Table 3.1에 제시하였다. 또한 비교를 위하여 평활모수로 $\lambda=0.2$ 와 0.4 를 사용 할 경우의 관리한계와 $\mathrm{EQL}$ 값 또한 수록하였다. 여기서 관리한계는 $\mathrm{ARL}_{0}=370.4,500,700$, 그리고 1000 을 만족하도록 설정하였다.

Table 3.1의 결과로부터 다음과 같은 사실을 알 수 있다. 첫째, 특정한 변화량을 예상하여 선정한 평활 모수 $\lambda$ 를 (예를 들면 0.2 또는 0.4 ) 사용하는 대신 제안된 절차에 의하여 최적의 평활모수를 선정하는 것이 $\mathrm{EQL}$, 즉 손실을 많이 줄일 수 있는 것으로 나타났다. 예를 들어 $\mathrm{EQL}$ 의 가장 큰 절감율을 나타낸 케이스는 $\mathrm{ARL}_{0}=1000$ 이고 $G(1,1)$ 사전분포를 가정하는 경우로서, $\lambda=0.4$ 를 사용하는 것에 비하여 최적의 평활모수인 $\lambda=0.11$ 을 사용하면 약 $32.95 \%$ 의 $\mathrm{EQL}$ 을 절감할 수 있었다. 둘째, 예상할 수 있었 던 바와 같이 작은 변화량에 많은 발생 확률을 배정하는 사전분포에서는 (예를 들면 $a=1$ 인 감마분포, 즉 지수분포(exponential distribution)) 최적의 평활모수가 작은 값을 가지며, 사전분포에서 중간과 큰 
Table 3.1. Optimal values of smoothing parameter, control limit, and EQL

\begin{tabular}{|c|c|c|c|c|c|c|c|c|}
\hline \multirow{2}{*}{ 사전분포 } & \multirow{2}{*}{$\mathrm{ARL}_{0}$} & \multicolumn{3}{|c|}{ 최적 설계 } & \multicolumn{2}{|c|}{$\lambda=0.2$} & \multicolumn{2}{|c|}{$\lambda=0.4$} \\
\hline & & $\lambda$ & $c$ & EQL & $c$ & EQL & $c$ & EQL \\
\hline \multirow{4}{*}{$G(1,1)$} & 370.4 & 0.16 & 2.815 & 11.68 & 2.859 & 11.77 & 2.959 & 13.99 \\
\hline & 500 & 0.14 & 2.893 & 12.54 & 2.962 & 12.78 & 3.054 & 15.88 \\
\hline & 700 & 0.13 & 2.994 & 13.54 & 3.073 & 14.05 & 3.157 & 18.45 \\
\hline & 1000 & 0.11 & 3.079 & 14.63 & 3.187 & 15.60 & 3.263 & 21.82 \\
\hline \multirow{4}{*}{$G(1,2)$} & 370.4 & 0.28 & 2.915 & 15.16 & 2.859 & 15.50 & 2.959 & 15.65 \\
\hline & 500 & 0.26 & 3.004 & 16.17 & 2.962 & 16.40 & 3.054 & 17.01 \\
\hline & 700 & 0.24 & 3.101 & 17.37 & 3.073 & 17.49 & 3.157 & 18.80 \\
\hline & 1000 & 0.22 & 3.200 & 18.73 & 3.187 & 18.75 & 3.263 & 21.08 \\
\hline \multirow{4}{*}{$G(1,3)$} & 370.4 & 0.33 & 2.937 & 15.88 & 2.859 & 16.72 & 2.959 & 16.01 \\
\hline & 500 & 0.31 & 3.027 & 16.81 & 2.962 & 17.55 & 3.054 & 17.08 \\
\hline & 700 & 0.29 & 3.125 & 17.94 & 3.073 & 18.54 & 3.157 & 18.49 \\
\hline & 1000 & 0.27 & 3.226 & 19.25 & 3.187 & 19.67 & 3.263 & 20.25 \\
\hline \multirow{4}{*}{$G(2,1)$} & 370.4 & 0.34 & 2.941 & 15.64 & 2.859 & 16.48 & 2.959 & 15.77 \\
\hline & 500 & 0.32 & 3.031 & 16.56 & 2.962 & 17.27 & 3.054 & 16.82 \\
\hline & 700 & 0.29 & 3.125 & 17.62 & 3.073 & 18.17 & 3.157 & 18.15 \\
\hline & 1000 & 0.27 & 3.226 & 18.79 & 3.187 & 19.17 & 3.263 & 19.76 \\
\hline \multirow{4}{*}{$G(2,2)$} & 370.4 & 0.48 & 2.975 & 17.62 & 2.859 & 20.23 & 2.959 & 17.75 \\
\hline & 500 & 0.46 & 3.065 & 18.30 & 2.962 & 21.00 & 3.054 & 18.39 \\
\hline & 700 & 0.44 & 3.165 & 19.12 & 3.073 & 21.87 & 3.157 & 19.16 \\
\hline & 1000 & 0.42 & 3.267 & 20.05 & 3.187 & 22.80 & 3.263 & 20.07 \\
\hline \multirow{4}{*}{$G(3,1)$} & 370.4 & 0.49 & 2.976 & 18.17 & 2.859 & 21.12 & 2.959 & 18.36 \\
\hline & 500 & 0.47 & 3.067 & 18.95 & 2.962 & 21.93 & 3.054 & 19.08 \\
\hline & 700 & 0.45 & 3.166 & 19.86 & 3.073 & 22.83 & 3.157 & 19.94 \\
\hline & 1000 & 0.43 & 3.268 & 20.89 & 3.187 & 23.78 & 3.263 & 20.92 \\
\hline \multirow{4}{*}{$U(0.25,7)$} & 370.4 & 0.45 & 2.970 & 23.15 & 2.859 & 25.91 & 2.959 & 23.21 \\
\hline & 500 & 0.43 & 3.060 & 23.98 & 2.962 & 26.89 & 3.054 & 24.02 \\
\hline & 700 & 0.41 & 3.159 & 25.02 & 3.073 & 28.04 & 3.157 & 25.03 \\
\hline & 1000 & 0.39 & 3.261 & 26.25 & 3.187 & 29.32 & 3.263 & 26.26 \\
\hline
\end{tabular}

변화량에 좀 더 많은 발생 확률을 배정할수록 최적의 평활모수의 값은 커짐을 알 수 있다. 셋째, 동일한 사전분포를 가정할 때 $\mathrm{ARL}_{0}$ 값을 크게 할수록 최적의 평활모수 값은 점점 작아지는 것을 알 수 있다.

\section{4. 결론}

이 논문에서는 정규분포를 따르는 공정에서 평균의 변화를 탐지하는 $\mathrm{EWMA}$ 관리도의 최적 설계에 대 한 절차를 제안하였다. 최적화를 위해 사용한 측도는 Reynolds와 Lou (2010)에서 사용한 EQL이며, 최적 설계는 이 측도를 최소로 하도록 평활모수를 선정하는 것이다. 이를 위하여 공정 평균의 변화량에 대한 사전분포로서 감마분포와 균등분포를 가정했으며, 다른 사전분포에 대해서도 동일한 절차를 적용 할 수 있을 것이다.

물론 변화량에 대하여 적절한 사전분포를 선정하는 것이 쉬운 일은 아니지만, 실무자들의 경험을 이용하 여 사전분포를 선정할 수 있다면 변화량을 예상하여 설정한 평활모수를 사용하는 것에 비하여 좀 더 효 율적으로 공정을 관리할 수 있을 것이라 생각한다. 


\section{References}

Capizzi, G. and Masarotto, G. (2003). An adaptive exponentially weighted moving average control chart, Technometrics, 45, 199-207.

Crowder, S. V. (1987a). A simple method for studying run length distributions of exponentially weighted moving average charts, Technometrics, 29, 401-407.

Crowder, S. V. (1987b). Average run lengths of exponentially weighted moving average control charts, Journal of Quality Technology, 19, 161-164.

Jiang, W., Shu, L. and Apley, D. W. (2008). Adaptive CUSUM procedures with EWMA-based shift estimators, IIE Transactions, 40, 992-1003.

Lucas, J. M. (1982). Combined Shewhart-CUSUM quality control schemes, Journal of Quality Technology, 14, 52-59.

Page, E. (1954). Continuous inspection schemes, Biometrika, 41, 100-115.

Reynolds, JR., M. R. and Lou, J. (2010). An evaluation of a GLR control chart for monitoring the process mean, Journal of Quality Technology, 42, 287-310.

Reynolds, JR., M. R. and Stoumbos, Z. G. (2004a). Control charts and the efficient allocation of sampling resources, Technometrics, 46, 200-214.

Reynolds, JR., M. R. and Stoumbos, Z. G. (2004b). Should observations be grouped for effective process monitoring?, Journal of Quality Technology, 36, 343-366.

Reynolds, JR., M. R. and Stoumbos, Z. G. (2006). Comparisons of some exponentially weighted moving average control charts for monitoring the process mean and variance, Technometrics, 48, 550-567.

Roberts, S. W. (1959). Control chart tests based on geometric moving averages, Technometrics, 1, 239-250.

Ryu, J.-H., Wan, H. and Kim, S. (2010). Optimal design of a CUSUM chart for a mean shift of unknown size, Journal of Quality Technology, 42, 311-326.

Shu, L. and Jiang, W. (2006). A Markov chain model for the adaptive CUSUM control chart, Journal of Quality Technology, 38, 135-147.

Sparks, R. S. (2000). CUSUM charts for signaling varying location shifts, Journal of Quality Technology, 32, 157-171.

Zhao, Y., Tsung, F. and Wang, Z. (2005). Dual CUSUM control schemes for detecting a range of mean shifts, IIE Transactions, 37, 1047-1057. 